\title{
The ethics of COVID-19 vaccine distribution
}

\author{
Ariadne A. Nichol ${ }^{1}$ (D) Kellen M. Mermin-Bunnell ${ }^{2}$
}

Accepted: 29 April 2021 / Published online: 19 May 2021

(c) The Author(s), under exclusive licence to Springer Nature Limited 2021

\section{Dear Editor,}

The COVID-19 pandemic has devastated the globe. As of 24 January, there are almost 100 million cases globally, over 25 million of which are in the United States [1]. Global deaths have passed 2 million and United States domestic deaths exceed 400,000. The Food and Drug Administration (FDA) approved for Emergency Use Authorization (EUA) the first safe and effective COVID-19 vaccine in the United States on December 12, 2020, the second on December 18, 2020, and the question now arises of how to distribute these vaccines. There are two ultimate goals of vaccination: prevention of infection and reduction in morbidity and mortality. Both can be achieved by vaccinating as many people as possible as soon as possible, and impact can be accelerated by prioritizing those most vulnerable to infection and to severe outcomes. However, numerous logistical barriers and a currently limited supply of COVID-19 vaccines prevent us from taking this rapid mass immunization approach. Because we cannot vaccinate everybody immediately, we should allocate the limited supply using a fair and just ethical framework.

In the United States, there are several differing vaccine allocation frameworks. The National Academies of Science, Engineering, and Medicine (NASEM) recommends first vaccinating high-risk healthcare workers and first responders and then people with comorbidities that put them at significantly higher risk, along with adults over 75 living in crowded settings [2]. The NASEM specifies the ethical and procedural principles underpinning their allocation framework as maximum benefit, equal concern, mitigation of health inequities, fairness, transparency, and evidence-based [2]. Notably, the framework did not include the principle of reciprocity, which is found in several other frameworks, including the World Health Organization's Strategic Advisory Group of Experts on Immunization (WHO SAGE) [3]. The NASEM accounted for the absence of the principle of reciprocity by citing that the principle is often too broadly defined and that there are other ways of incorporating

Ariadne A. Nichol

ariadnen@stanford.edu

1 Center for Biomedical Ethics, Stanford University School of Medicine, 1215 Welch Road, Modular A, Stanford, CA 94305, USA

2 Yale University, Connecticut, USA 
essential social roles into the framework. The NASEM principles mirror those of the Centers for Disease Control and Prevention's (CDC) Advisory Committee on Immunization Practices (ACIP) [4]. The ACIP put forth a framework that recommends allocating vaccines to the following priority groups in Phase One: (a) healthcare workers and long-term care facilities' residents, (b) essential workers, and (c) adults over 65 or those with high-risk medical conditions [4]. The ethical principles underlying this framework are transparency, maximization of benefits and minimization of harms, promotion of justice, and mitigation of health inequities [4].

Both the CDC and the NASEM frameworks address the current inequities in morbidity and mortality between African American, Latinx, American Indian and Alaska Native, and Native Hawaiian and Pacific Islander communities and white individuals in the United States [2, 4]. The national-level recommendations are now up for interpretation and incorporation by individual states, and we have seen variation in how states are tackling COVID-19 vaccine allocation and the level of granularity in the publicly available plans. For example, Tennessee has incorporated CDC's Social Vulnerability Index (SVI) into their allocation framework to promote equity, and $5 \%$ of the state's Moderna vaccine allocation will go to counties with a high SVI value [5]. Yet, there is still a degree of uncertainty for the public on how, when, and to whom the vaccines will be allocated, in part because there are many moving parts in allocating vaccines during this pandemic. Acknowledging that changes will have to be made to distribution protocols as more information comes out, transparency in developing and revising frameworks will remain critical. We need coordinated efforts and strong national leadership to be successful in launching a vaccine campaign that is accepted as fair and is viewed as trustworthy by the public.

Due to the lack of strict and specific national recommendations, each regional vaccine distribution center has flexibility to determine its own distribution framework. This leaves much room for error. Some hospitals have chosen to use an algorithm. For example, vaccine priority $=$ risk of death $\times$ risk of exposure $\times$ risk of transmitting to others. However, using artificial intelligence to implement an algorithm such as this one poses its own challenges. At Stanford Hospital, only 7 out of 5000 initially available vaccines were allocated to residents, who, along with nurses, are the primary caretakers of patients and thus are at highest risk of infection [6]. Stanford has since developed a new algorithm, exemplifying the necessary practice of continual reevaluation of protocol as data are collected.

Identifying the most vulnerable groups in the pandemic so that they may be prioritized in vaccine distribution can help shape our response not just within one country, but also globally. Vaccine nationalism poses a significant threat to eradicating COVID-19, by undermining the recognition of all people's dignity and right to health beyond national borders. Moreover, the sentiment neglects the reality of our increasingly interconnected world. A passenger can travel in a matter of hours from one part of the world to another, demonstrating the ease with which an infectious disease can spread globally. Therefore, in evoking a principle of solidarity, we should put our efforts towards working to establish a global vaccination campaign in order to protect vulnerable priority groups and diminish the effects of COVID-19 for all. 
Several entities have proposed strategic frameworks to equitably allocate limited supplies of vaccines globally. The World Health Organization (WHO) Strategic Advisory Group of Experts on Immunization (SAGE) has put forth recommendations that include six guiding principles of human well-being, equal respect, global equity, national equity, reciprocity, and legitimacy [3]. The principle of global equity is meant to ensure that countries unable to secure vaccines, such as low- and middleincome countries (LMICs), are able to adequately provide vaccines to their populations by having other countries with financial means, at minimum, reduce barriers for obtaining vaccines; or as we would recommend, contribute vaccines for people in those countries. Another global COVID-19 vaccine allocation framework known as the Fair Priority Model was proposed by E. Emanuel et al. [7]. The framework takes a calculated approach to vaccine allocation, utilizing Standard Expected Life Years Lost (SELYL). The underlying ethical principles are benefiting people and limiting harm, prioritizing the disadvantaged, and equal moral concern [7].

In all of these frameworks, one thing remains crystal clear: uncertainty shrouding the evolving pandemic should not deter us from creating flexible, yet fair evidencebased frameworks for the COVID-19 response. We need international support and collaboration to establish a global supply chain that will serve those with high risk of morbidity and mortality or high risk of infection regardless of country of origin. We also need active campaigns, perhaps by taking advantage of widely used social media platforms, to combat the spread of misinformation surrounding COVID-19 and the vaccines available.

Friction remains between allocation recommendations established using ethical frameworks in a theoretical manner and the lived reality of vaccine distribution on the ground. In the United States, a limited but substantial supply of over 41 million vaccine doses have been distributed to states, yet only roughly 18 million people had been vaccinated as of 20 January [8]. This gap between distribution and vaccination is concerning since both the Pfizer-BioNTech and the Moderna mRNA vaccines must be kept at cold temperatures, and if they sit at room temperature for too long they must be discarded. As the pandemic rages across the country, the dire need for vaccines exists in stark contrast with the disposal of unused doses. The question arises as to whether the United States should continue to follow allocation guidelines precisely, knowing that vaccines will be wasted, or use the leftover doses, and by necessity, vaccinate some on a first come, first served basis. Some may be inclined to vaccinate as many people as possible as quickly as possible, but this freefor-all approach could not only end up being highly unethical in light of the carefully developed frameworks, but also may reduce the public's trust in our healthcare systems and potentially increase vaccine hesitancy. This is a concern especially for the mitigation of racial health inequities, as Black, Latinx, and Native Americans have been hit hardest by the pandemic and have high rates of vaccine hesitancy due to historical, unethical medical experimentation [9].

Given this complex logistical situation, there could be lists to call up of those in high-priority groups based on risk of mortality and risk of exposure. There could also be mobile vaccination units to take the unused doses and get them into the arms of those that may otherwise not be able to access vaccinations. For example, the United States Federal Emergency Management Agency (FEMA) has provided 
mobile vaccination vans in multiple regions throughout the country to specifically target regions based on the CDC's SVI criteria to increase vaccination accessibility among vulnerable populations [10]. Such measures should be enacted and performed by the relevant government public health authorities to increase public trust and standardize logistical decision-making.

We believe COVID-19 will prove to be a generation defining event. As we make decisions of how we allocate vaccines to our population, both locally and abroad, we are also creating a standard for future emerging infectious disease pandemics. Therefore, it is critical to approach these decisions through an ethical lens that allows us to distinguish an equitable way to move forward and continue to ensure the public's trust in our healthcare systems.

\section{Declarations}

Conflict of interest On behalf of all authors, the corresponding author states that there is no conflict of interest.

\section{References}

1. Johns Hopkins University \& Medicine. Coronavirus Resource Center. https://coronavirus.jhu.edu. Accessed 24 January 2021.

2. Gayle H, Foege W, Brown L, Kahn B, editors. Framework for Equitable Allocation of COVID-19 Vaccine. Washington, DC: National Academies Press; 2020.

3. World Health Organization. WHO SAGE values framework for the allocation and prioritization of COVID-19 vaccination. 2020.

4. McClung N, Chamberland M, Kinlaw K, Bowen Matthew D, Wallace M, Bell BP, et al. The Advisory Committee on Immunization Practices' Ethical Principles for Allocating Initial Supplies of COVID-19 Vaccine-United States, 2020. MMWR Morb Mortal Wkly Rep. 2020;69(47):1782-6.

5. Tennessee Department of Health. COVID-19 Vaccination Plan Tennessee. 2020. https://www.tn. gov/content/dam/tn/health/documents/cedep/novel-coronavirus/COVID-19_Vaccination_Plan.pdf. Accessed 24 January 2021.

6. Wu KJ, Isaac M. Frontline workers were left off the vaccine list at Stanford Medical Center in Palo Alto. They fought back. The New York Times. 2020; https://www.nytimes.com/2020/12/18/world/ covid-stanford-health-center-vaccine-protest.html. Accessed 24 January 2021.

7. Emanuel EJ, Persad G, Kern A, Buchanan A, Fabre C, Halliday D, et al. An ethical framework for global vaccine allocation. Science. 2020;369(6509):1309-12. https://doi.org/10.1126/science.abe28 03.

8. Centers for Disease Control and Prevention. Trends in Number of COVID-19 Vaccinations in the US. COVID Data Tracker. 2021; https://covid.cdc.gov/covid-data-tracker/\#vaccination-trends. Accessed 24 January 2021.

9. Coustasse A, Kimble C, Maxik K. COVID-19 and Vaccine Hesitancy. J Ambul Care Manag. 2021;44(1):71-5. https://doi.org/10.1097/JAC.0000000000000360.

10. Federal Emergency Management Agency. Mobile Vaccination Centers Improve Vaccine Accessibility. 2021.

Publisher's Note Springer Nature remains neutral with regard to jurisdictional claims in published maps and institutional affiliations. 